\title{
Vascular Defects and Sensorineural Deafness in a Mouse Model of Norrie Disease
}

\author{
Heidi L. Rehm, ${ }^{1,2,3 *}$ Duan-Sun Zhang, ${ }^{1,3 *}$ M. Christian Brown, ${ }^{2,4}$ Barbara Burgess, ${ }^{4}$ Chris Halpin, ${ }^{2,4}$ \\ Wolfgang Berger, ${ }^{5}$ Cynthia C. Morton, ${ }^{2,6}$ David P. Corey, ${ }^{1,2,3}$ and Zheng-Yi Chen ${ }^{2,7}$ \\ ${ }^{1}$ Neurosurgery Service, Massachusetts General Hospital, Boston, Massachusetts 02114, 2Harvard Medical School, \\ Boston, Massachusetts 02115, 3Howard Hughes Medical Institute, Boston, Massachusetts 02114, ${ }^{4}$ Department of \\ Otology and Laryngology, Massachusetts Eye and Ear Infirmary, Boston, Massachusetts 02114, 5Max Planck Institute for \\ Molecular Genetics, 14195 Berlin, Germany, ${ }^{6}$ Departments of Obstetrics, Gynecology, and Reproductive Biology and \\ Pathology, Brigham and Women's Hospital, Boston, Massachusetts 02115, and 7Neurology Service, Massachusetts \\ General Hospital, Boston, Massachusetts 02114
}

Norrie disease is an X-linked recessive syndrome of blindness, deafness, and mental retardation. A knock-out mouse model with an Ndp gene disruption was studied. We examined the hearing phenotype, including audiological, histological, and vascular evaluations. As is seen in humans, the mice had progressive hearing loss leading to profound deafness. The primary lesion was localized to the stria vascularis, which houses the main vasculature of the cochlea. Fluorescent dyes

Norrie disease is an $\mathrm{X}$-linked recessive neurological syndrome that has been characterized extensively by Warburg (1961, 1963, 1965, 1966). The characteristic feature is congenital bilateral blindness with a prominent intraocular mass (pseudoglioma). An additional ocular phenotype of note is a partial avascularity of the retina. Most, if not all, patients manifest hearing loss (Parving and Warburg, 1977; C. Halpin and H. L. Rehm, unpublished observations), although its onset may range from 4 months to 45 years (Gorlin et al., 1995). Also, mental retardation and/or psychotic features develop in approximately two-thirds of patients (Gorlin et al., 1995).

Since the Norrie disease gene, NDP (Norrie disease, pseudoglioma), was cloned (Berger et al., 1992; Chen et al., 1992), $>80$ different mutations have been found (Online Mendelian Inheritance in Man, 2001). In addition, the X-linked form of familial exudative vitreoretinopathy (FEVR) was found to be caused by missense mutations in NDP (Chen et al., 1993; Fuchs et al., 1995; Meindl et al., 1995; Torrente et al., 1997; Shastry et al., 1997; Shastry, 1998). Many patients with X-linked FEVR report no vision problems, although all have some degree of retinal avascularity and a significant portion have more severe pathology (Guyer et al., 1999).

\footnotetext{
Received Dec. 26, 2001; revised Feb. 21, 2002; accepted March 12, 2002.

This work was supported by the Howard Hughes Medical Institute and by National Institutes of Health Grants DC02281 (D.P.C.), DC03402 (C.C.M.), and DC04546 (Z.-Y.C.). H.L.R. is an Associate and D.P.C. is an Investigator of the Howard Hughes Medical Institute. We thank J. Adams for his technical assistance with mouse perfusions and for his help in interpreting the histopathology in the inner ear.

*H.L.R. and D.-S.Z. contributed equally to this work.

Correspondence should be addressed to Dr. Zheng-Yi Chen, WEL425, Neurology Department, Massachusetts General Hospital, 55 Fruit Street, Boston, MA 02114, E-mail: zhengyi@helix.mgh.harvard.edu, or to Dr. David P. Corey, WEL415, Howard Hughes Medical Institute, Massachusetts General Hospital, 50 Blossom Street, Boston, MA 02114, E-mail: corey@helix.mgh.harvard.edu.

Copyright (C) 2002 Society for Neuroscience $0270-6474 / 02 / 224286-07 \$ 15.00 / 0$
}

showed an abnormal vasculature in this region and eventual loss of two-thirds of the vessels. We propose that one of the principal functions of norrin in the ear is to regulate the interaction of the cochlea with its vasculature.

Key words: Norrie disease; mouse model; deafness; blindness; retina; cochlea; stria vascularis; marginal cells; vascular; angiogenesis; norrin

$N D P$ encodes a small secreted protein belonging to the cysteine knot growth factor family (Meindl et al., 1992; Meitinger et al., 1993), which is expressed in the brain and the retina, but not in the heart, spleen, lung, muscle, liver, kidney, or testis (Berger et al., 1992; Chen et al., 1992). By in situ hybridization, Ndp expression begins after embryonic day 18 (in mice) and is found in the neural layers of the retina, throughout the brain, and in the spiral ganglion and stria vascularis of the cochlea (Berger et al., 1996; Z.-Y. Chen, unpublished observations).

A knock-out mouse model of Norrie disease shows fibrous masses in the vitreous body of the eye as well as consistent disorganization of the ganglion cells of the retina and sporadic disorganization or degeneration of other cells in the retina (Berger et al., 1996). The retinal vasculature in mice with Norrie disease is abnormal by postnatal day 9 (P9), showing abnormal vessels in the inner retina and few vessels in the outer retina (Richter et al., 1998).

$N d p$ knock-out mice have an eye phenotype that resembles that in human Norrie disease, suggesting that this mouse may be a good model to study other abnormalities associated with the disease. Therefore, we examined the hearing phenotype using audiology, histology, and vascular perfusion techniques. We found that knock-out mice had progressive hearing loss beginning at 3 months of age, whereas controls retained normal hearing to at least 15 months of age. We also showed that this hearing loss is very similar to Norrie disease in humans. Histology of cochleas from the knock-out mice revealed that the earliest primary site of cellular pathology occurs in the stria vascularis, with other regions affected secondarily. Histology and fluorescent dye fills of vasculature showed an abnormal variation in vessel size in knockout mice and loss of more than one-half of the vasculature as the hearing loss progressed. These observations suggest that one of 
the principal functions of norrin is to regulate interaction of the cochlea with its vasculature.

\section{MATERIALS AND METHODS}

Animals. Norrie disease mice were generated by targeted deletion of the $N d p$ locus as described previously (Berger et al., 1996). The original $N d p$ mutation was made in the 129/Ola mouse strain and then backcrossed to C57BL/6 for five generations. This strain was then out-crossed to $129 / \mathrm{Sv}$ for two generations for initial analyses. Subsequently, mice were outcrossed once into the CD1 strain (129/CD1) and then out-crossed twice into the $\mathrm{CBA} / \mathrm{CaJ}$ background. This later strain was used because it has much less age-related hearing loss than others (Zheng et al., 1999). The results reported here are from the $\mathrm{CBA} / \mathrm{CaJ}$ strain. In all experiments, wild-type controls were littermates of the $N d p$ knock-out mice. Genotyping was performed by PCR of DNA extracted from tail biopsies, as described previously (Berger et al., 1996).

Mouse auditory tests. Auditory brainstem responses (ABRs) were measured using a closed-field, calibrated sound system and measured responses to tone bursts (Fig. 1A); in this case, thresholds are specified in terms of decibels sound pressure level (dB SPL). For both methods, mice were anesthetized with ketamine $(100 \mathrm{mg} / \mathrm{kg}$, i.p.) and xylazine (Rompun; $10 \mathrm{mg} / \mathrm{kg}$, i.p.) and kept in a heated chamber. The left ear was tested. No surgery was performed except occasionally when the left tragus was cut to increase access to the ear canal. The frequencies tested included 5.7, 8, 11.3, 16, 22.6, 32, and $45.3 \mathrm{kHz}$. Stimuli were of alternating polarity and repeated at $40 \mathrm{sec}^{-1}$. Tone bursts were of $10 \mathrm{msec}$ duration with a $0.5 \mathrm{msec}$ rise-fall time. Stimulus levels were spaced $10 \mathrm{~dB}$ apart. Responses were filtered and amplified 10,000 times. At each sound level, 128 responses were averaged.

Vestibular tests. Mice were placed into a water tank to assess their ability to swim and stay upright. Brn-3.1 knock-out mice (which lack functional hair cells) (Xiang et al., 1996) were used as positive controls; wild-type littermates were used as negative controls.

Histology. Mice were anesthetized with urethane (1.5 gm/ $/ \mathrm{kg}$, i.p.) and perfused intracardially with $5 \mathrm{ml}$ of PBS $/ 0.1 \%$ sodium nitrite followed by $5 \mathrm{ml}$ of $4 \%$ paraformaldehyde. Cochleas were dissected and then fixed and decalcified in $10 \%$ formalin/0.14 M EDTA for $4-5 \mathrm{~d}$ with a daily change of solution. The tissues were then treated with osmium tetroxide, dehydrated in graded alcohols, equilibrated in propylene oxide, and embedded in Epon (Electron Microscopy Sciences, Fort Washington, PA). The embedded cochleas were sectioned at $2 \mu \mathrm{m}$ and stained with $1 \%$ toluidine blue.

Vasculature analysis. Mice were anesthetized by injection with urethane $(1.5 \mathrm{gm} / \mathrm{kg}$, i.p.) and perfused intracardially with $4 \mathrm{ml}$ of $10 \mathrm{mg} / \mathrm{ml}$ FITC-labeled dextran (Sigma, St. Louis, MO). Cochleas were dissected, fixed in $4 \%$ paraformaldehyde, and decalcified as described above. Cochleas were hemisectioned by cutting perpendicular to the axis of the modiolus between the base and apical turn. Each half was mounted for fluorescence imaging. The eyes were also dissected; the corneas were removed and the retinas were separated from the choroid. Four cuts were made in each retina to allow it to lay flat. Serial confocal images were taken every $5 \mu \mathrm{m}$ over the entire thickness of the retina $(125 \mu \mathrm{m})$. The images were separated into two groups and then merged to form two final images for each retina, as depicted in Figure $6 G-J$ (the top 13 sections were merged to represent the inner retina and the bottom 13 sections were merged to represent the outer retina). The images shown in Figure 6 are limited to the central portion of the retina; however, similar observations were seen in the periphery of the retina (data not shown). Imaging was performed using either a Radiance 2000 Confocal Imaging System (Bio-Rad, Hercules, CA) (see Fig. $6 E-J$ ) or a MZ FLIII fluorescence stereomicroscope (Leica, Nussloch, Germany) (see Fig. $6 A-D)$.

Human auditory tests. The human audiogram (Fig. 2) was obtained using standard audiometry for pure tones (American National Standards Institute S3.21 1978, R-1986). Informed consent was obtained (Massachusetts Eye and Ear Infirmary Institutional Review Board protocol \#93-05-16).

\section{RESULTS}

\section{Auditory tests}

ABRs were recorded from $N d p$ knock-out mice and littermate controls (CBA/CaJ strain) at three ages: 3-4 months, 6-8 months, and 13-15 months (Fig. 1A). Although wild-type controls
A
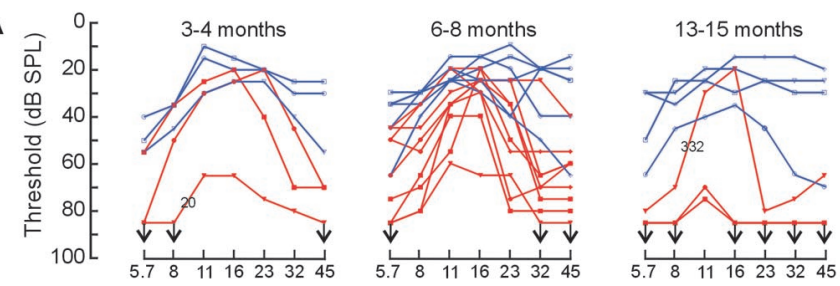

B

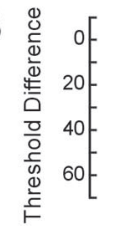
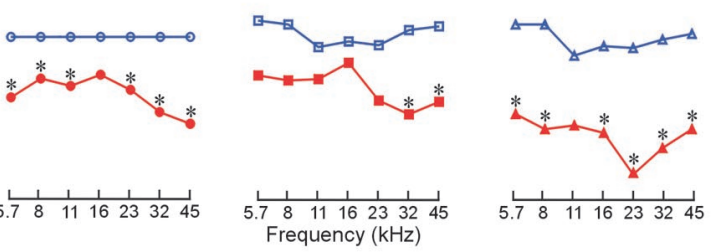

C

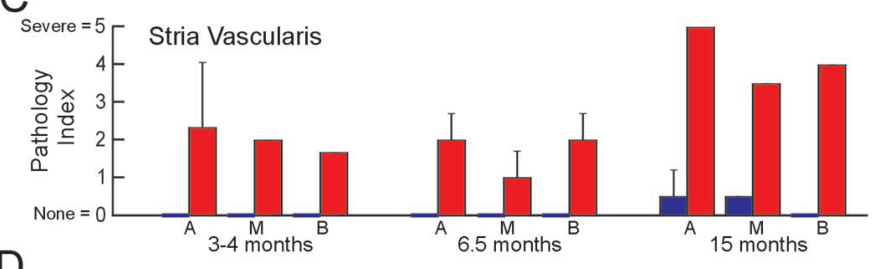

$\mathrm{D}_{\mathrm{s}}$
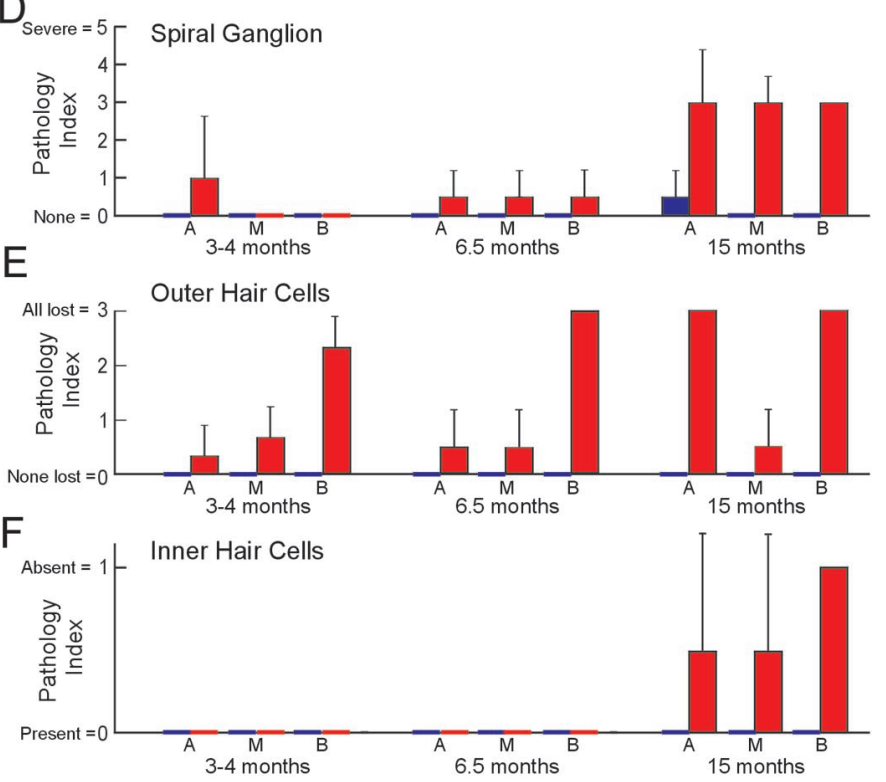

Figure 1. Audiology aligned with histopathological analysis. $A$, ABRs to pure tone bursts in knock-out (red) and control (blue) mice, separated into three age groups. Thresholds are plotted with highest sensitivity (lowest SPL) upward on the graphs for comparison with the human audiogram shown in Figure 2. Arrows indicate no response to the highest sound pressure level tested ( $85 \mathrm{~dB}$ SPL). $B$, Difference in average threshold shifts compared with a 3-4 month control average. Asterisks indicate statistically significant differences at the indicated frequency. $C-F$, Pathology in indicated regions of the cochlea in knock-out (red) and control (blue) mice, separated into three age groups. The system for rating pathology in the inner and outer hair cells and the spiral ganglion was based on the quantity of hair cells or neurons lost. The system for rating pathology in the stria vascularis was based on the presence of the various types of pathology described in Figure 4. Ratings for each mouse in each region were averaged to get an overall rating for the group. Bars indicate $1 \mathrm{SD}$. The significance of separating the pathology into three regions is attributable to the tonotopic organization of the cochlea, with low frequencies detected in the apex $(A)$ of the cochlea, middle frequencies in the middle $(M)$, and high frequencies in the base $(B)$. This allows comparison of the audiological data with the histopathology. 


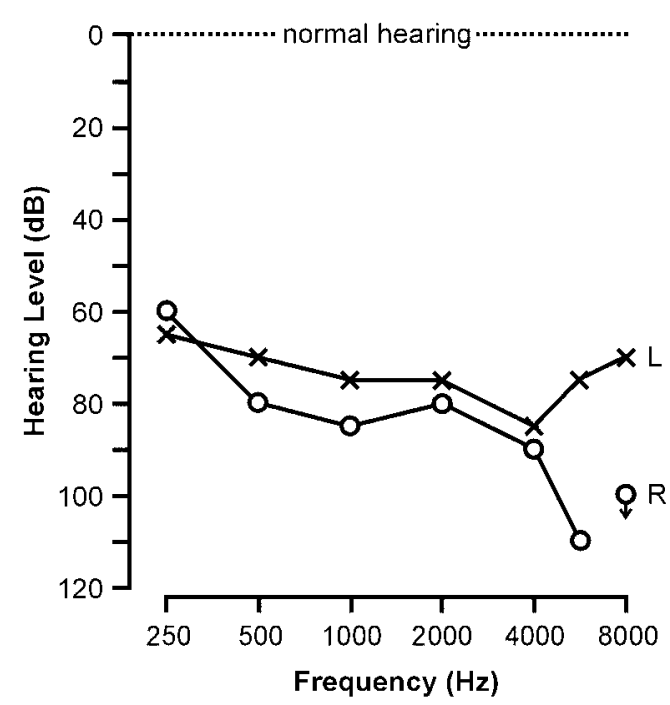

Figure 2. Audiogram of a 40-year-old patient with Norrie disease. Symbols ( $\times$, left ear; $\bigcirc$, right ear) represent threshold response levels for the tones at the indicated frequencies relative to normal. There is a severe bilateral sensorineural hearing loss with elevated threshold (lower sensitivity) for sounds at all frequencies. The arrow indicates no response at the highest sound level tested.

maintained a relatively stable level of hearing at all ages, the knock-out mice showed a progressive hearing loss across all frequencies (Fig. 1B). Hearing loss was initially more severe in the high frequencies but eventually progressed to a relatively flat, profound loss by 15 months. Exceptions were one knock-out mouse with substantial hearing loss at 3.2 months (Fig. $1 A$, mouse $20)$ and one that retained hearing in the middle frequencies at 13 months (Fig. $1 A$, mouse 332).

We found similar progressive hearing loss in $N d p$ knock-out mice on a $129 /$ Sv background. However, the natural hearing loss in this strain (Zheng et al., 1999) masked differences between wild-type and knock-out animals until the age of 15 months, when the knock-out mice become profoundly deaf (data not shown).

The hearing loss in the $N d p$ knock-out mice is very similar to that in human patients with Norrie disease. Figure 2 shows the audiogram of an affected 40-year-old individual, typical of that age, which reveals severe hearing loss elevating thresholds at all frequencies in both ears. As in the knock-out mice, there is a slightly higher loss at higher frequencies.

\section{Vestibular tests}

No behavioral signs indicative of vestibular defects, such as circling or head shaking, have been observed in $N d p$ knock-out mice. To look for more subtle defects in vestibular function, a swim test was performed. Although Brn-3.1 knock-out mice (which lack functional hair cells because of the missing transcription factor) (Xiang et al., 1996) tumbled in the water, unable to swim or stay upright, the $N d p$ knock-out and control mice were able to swim normally, confirming normal function of the vestibular system.

\section{Histological analysis of the cochlea}

A subset of mice was killed immediately after their ABR thresholds were measured. Toluidine-blue-stained plastic sections from the inner ears of the $N d p$ knock-out mice were compared with those from littermate controls. In the controls, all cochlear structures were preserved at all three ages (Fig. 3A,C) (6.5 months; data not shown). However, we found pathological changes in the knock-out mice that were consistent with the audiological deficits. These changes were first observed at 3 months. It should be noted that cochleas from knock-out mice at P12 appeared normal by histological analysis (data not shown).

To measure the pathological changes and determine which are primary and which may be secondary to other degeneration, the pathology was quantified. In all cochlear structures in every region (apex, middle, and base) of a middle section of the modiolus, changes were rated subjectively. From this analysis (Fig. $1 A-D)$, it is clear that the stria vascularis is the structure most affected in all age groups. Significantly enlarged vessels were observed in the stria vascularis, particularly in the apex of the cochlea (Figs. 3B, 4B), as were a variety of other pathological changes (Fig. $4 C-F$ ). A gradual loss of outer hair cells was observed, which is particularly consistent with auditory threshold increases. As the hearing loss progressed, the spiral ganglion began to degenerate: there was slight neuronal loss in mice aged 6.5 months and substantial loss by 15 months (Figs. 1D, 2D). Although inner hair cells in all turns were well preserved at the beginning of hearing loss, eventual loss of the inner hair cells occurred in advanced stages (Fig. 1E,F).

One exception to the trends described above was the presence of spiral ganglion pathology in the apex of a knock-out mouse at only 3 months. However, this can be explained by the much more advanced hearing loss in this young mouse (Fig. 1 $A$, mouse 20).

Although most cochlear pathology consisted of cell loss, additional differences were observed in the stria vascularis of knockout animals (Fig. 4). Large vessels were consistently observed, often with multiple endothelial cell nuclei per capillary cross section (Fig. 4B, arrows). Intercellular spaces observed in many sections (Fig. 4C,D) were uncharacteristic of healthy cochleas (Fig. 4A). An increase in nuclei in the intermediate cell layer in some areas (Fig. 4C, asterisks) as well as a complete loss of marginal cells in others (Fig. $4 E$ ) were also seen in knock-out mice. In severe cases, the stria vascularis was almost completely degenerated and often accompanied by fibrocyte loss in the adjacent spiral ligament (Fig. $4 F$ ).

Despite the loss of nerve fibers and cell bodies in the spiral ganglion of the cochlea, innervation of the adjacent vestibular organs remained intact (data not shown), consistent with the behavioral tests that indicate normal vestibular function.

\section{Cochlear and retinal vasculature studies}

Because enlargement of the vessels of the stria vascularis was one of the earliest abnormalities in the knock-out mouse, we measured vessels in detail. The cross-sectional diameter and number of vessel cross sections per turn were tallied for all turns in each mouse aged 3-4 months (Fig. 5). We found substantially more vessels with cross sections of $>10 \mu \mathrm{m}$ (Fig. 5); the average vessel size was significantly larger $(p<0.005)$ in the knock-out mice $[7.4 \pm 6.1 \mu \mathrm{m}($ mean $\pm \mathrm{SD})]$ than in controls $(4.5 \pm 1.9 \mu \mathrm{m})$. However, the average vessel sizes of the two older groups of knock-out mice were not significantly different from those of the 3- to 4-month-old controls. This age-related loss of larger vessels may be part of the more general loss of vessels in the knock-out mice. Although the number of vessels per cochlear turn is similar in knock-out and control mice at 3-4 months of age, almost two-thirds of vessels were lost in 15-month-old knock-out mice $(p<0.005)$ (Fig. 5, inset) .

To differentiate a dilation mechanism of vessel enlargement (which could be artificially induced) as opposed to a more per- 

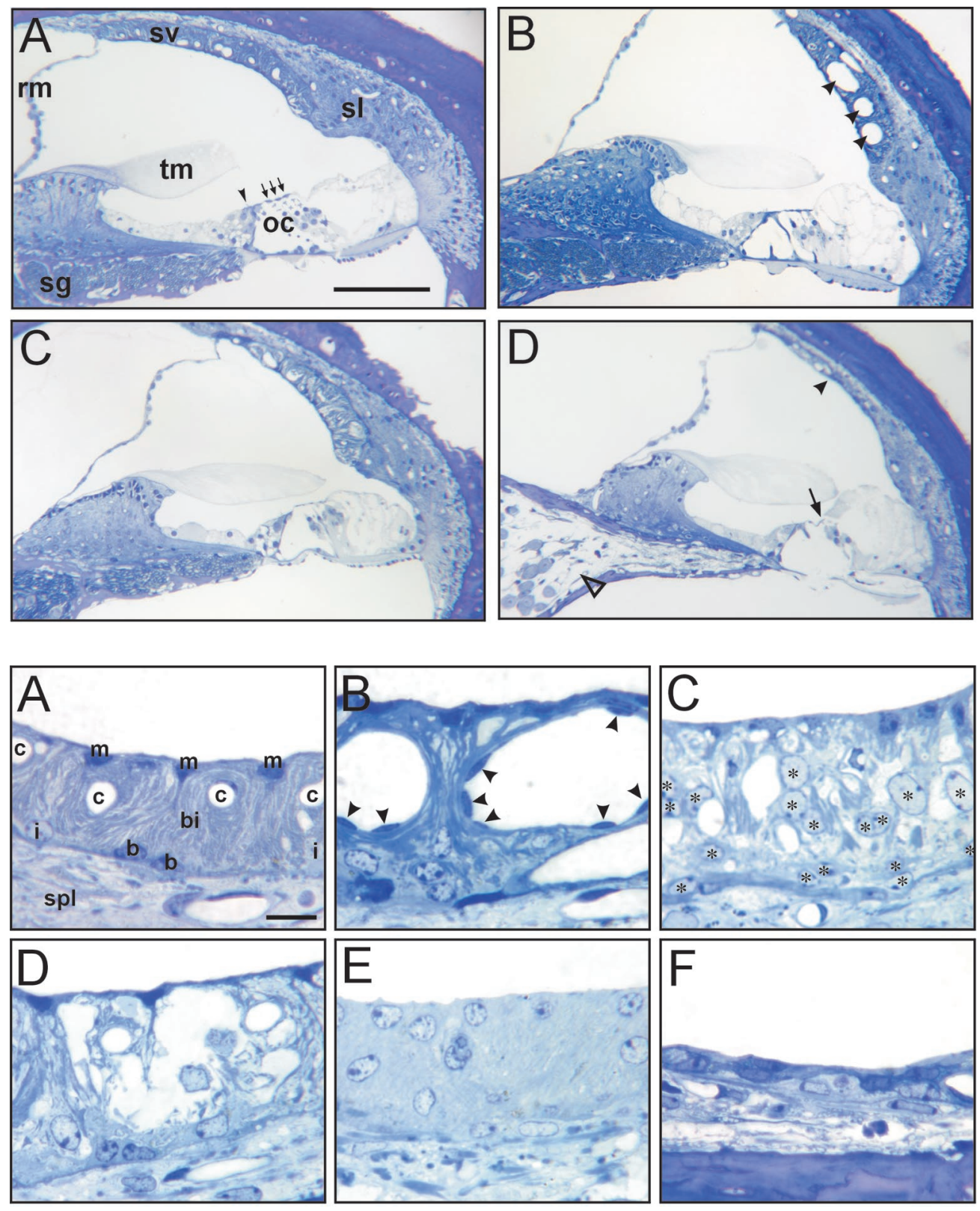

Figure 3. Histology of knock-out and control mouse cochleas. $A$, A normal cochlea from the apical turn of a 3.2month-old control mouse. The arrowhead indicates an inner hair cell; arrows indicate outer hair cells. $B$, Abnormal cochlea from the apical turn of a 3.2month-old knock-out mouse. Note the enlarged vessels (arrowheads). C, Normal cochlea from the apical turn of a 15 -month-old control mouse. $D$, Abnormal cochlea from the apical turn of a 15 -month-old knock-out mouse. Note the degenerated stria vascularis (filled arrowhead), degenerated spiral ganglion (open arrowhead), and loss of outer hair cells (arrow). oc, Organ of Corti; rm, Reissner's membrane; sg, spiral ganglion; $s l$, spiral ligament; $s v$, stria vascularis; $t m$, tectorial membrane. Scale bar, $100 \mu \mathrm{m}$.

Figure 4. Histology of the stria vascularis. $A, \mathrm{~A}$ normal stria vascularis from the basal turn of a 15-month-old control mouse. A normal distribution of nuclei from basal $(b)$, intermediate $(i)$, and marginal $(m)$ cells is present. Normal basal infoldings $(b i)$ extending from the marginal and intermediate cells and surrounding the capillaries $(c)$ are seen. The adjacent spiral ligament $(s p l)$ is also shown. $B$, Stria from the apical turn of a 3.2-month-old knock-out mouse showing enlarged vessels (arrowheads indicate endothelial cell nuclei). $C$, Stria from the basal turn of a 3.2-month-old knock-out mouse showing excessive numbers of cells in the intermediate cell layer (asterisks indicate nuclei). The origin of these cells is not known. $D$, Stria from the middle region of the cochlea of a 6.5-month-old knock-out mouse showing cell loss and replacement by large intracellular spaces. $E$, Stria from the basal turn of a 15-month-old knock-out mouse showing a complete loss of marginal cells (top). F, Stria from the apical turn of a 15-month-old knock-out mouse showing severe degeneration of the whole structure. Scale bar, $10 \mu \mathrm{m}$.

manent structural difference, each capillary cross section of $\geq 13$ $\mu \mathrm{m}$ was scored for the number of nuclei and compared with capillaries of $<13 \mu \mathrm{m}$ from the control and knock-out mice. The average number present in the large vessels of the knock-out mice was $1.8 \pm 1.4$, significantly more than in the normal-sized vessels of the control mice $(0.68 \pm 0.56 ; p=0.01)$ or knock-out mice $(0.52 \pm 0.58 ; p=0.0025)$.

To better assess the entire vasculature of the cochlea, mice were perfused with FITC-labeled dextran and whole turns were visualized using confocal microscopy. The large vessels observed in histological sections were also observed through this method, as were a general disorganization of blood vessels and the lack of a well developed capillary bed (Fig. 6B,D,F). These changes were particularly pronounced in the apical portion of the cochlea (Fig. $6 B, F$ ), consistent with the histo- logical data. Although large vessels were not seen in the spiral ganglion vasculature, this region did show a poorly developed capillary bed (Fig. $6 F$ ).

In addition to the ages examined for histology and hearing loss (3-4, 6-8, and 13-15 months), perfusions were also performed on mice as young as 1.5 months of age. Although some enlarged vessels were seen at this age in the knock-out mice, the changes were much less substantial compared with those observed at 23-4 months (data not shown). This is consistent with the normal histology present at P12 and may indicate a degenerative pathological process that occurs after normal development of the cochlea.

For comparison with the cochlear vascular phenotype, we also examined the retinas of the FITC-perfused mice. Some similar changes were seen, including a lack of a well developed fine 


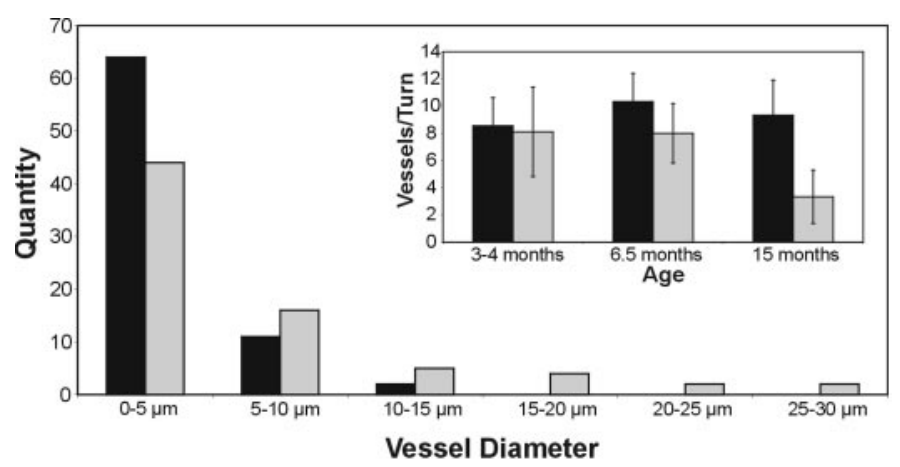

Figure 5. Comparison of vessel size. The distribution of cross-sectional vessel diameters for control (black) and knock-out (gray) mice at 3-4 months is shown. The inset shows the average number of vessels per turn in control (black) and knock-out (gray) mice, separated by age. Bars indicate $1 \mathrm{SD}$.

capillary network in both the inner and outer retina layers (Fig. $6 G-J)$. Particularly striking was the absence of any vascular development in the outer retina of the knock-out mice (Fig. 6I,J).

\section{DISCUSSION}

We have demonstrated progressive hearing loss leading to profound deafness in $N d p$ knock-out mice. The loss is similar to that in human patients with Norrie disease; it is progressive, affects all frequencies, and is more severe in the high frequencies. Although there is some variability in the severity and age of onset, the penetrance appears to be complete. Early descriptions of the human Norrie disease clinical phenotype document a much lower incidence, with only one-third of patients expressing hearing loss. Although this difference may result from the lack of genetic variation in an inbred mouse strain, there are several other possible explanations. First, many studies were based on selfreporting of hearing status without audiological testing, possibly missing milder hearing losses. Second, because the onset of the hearing loss ranges from 4 months to 45 years (Gorlin et al., 1995), many patients may not have been followed long enough to detect this phenotype. In a study of 53 patients with Norrie disease from 14 families, 32 were tested audiometrically and hearing loss was found in 27 (Parving and Warburg, 1977). The five with normal hearing were all under age 20. Furthermore, a recent study of two groups of patients from the United States and Costa Rica showed hearing loss in every individual with Norrie disease who was tested, except for a child 2 years of age (Halpin and Rehm, unpublished observations). Thus, the human audiological phenotype is much more frequent than reported previously, and the high penetrance and progressive nature of the hearing loss in the knock-out mice is consistent with the human disease.

There are limited data on hearing function and pathology from patients with Norrie disease. In an electrophysiological study of three patients with hearing loss (ages 22, 27, and 30 years) (Parving et al., 1978), measurements of cochlear microphonics (testing hair-cell function) and brainstem potentials (testing auditory brainstem function) were normal, whereas whole-nerve action potentials (testing spiral ganglion and cochlear nerve function) were abnormal. This analysis suggests a defect in the spiral ganglion neurons with initial preservation of hair cells but does not rule out an additional lesion in the stria vascularis. In addition, the only temporal bone study documented was performed postmortem on a 77-year-old patient with Norrie disease who had
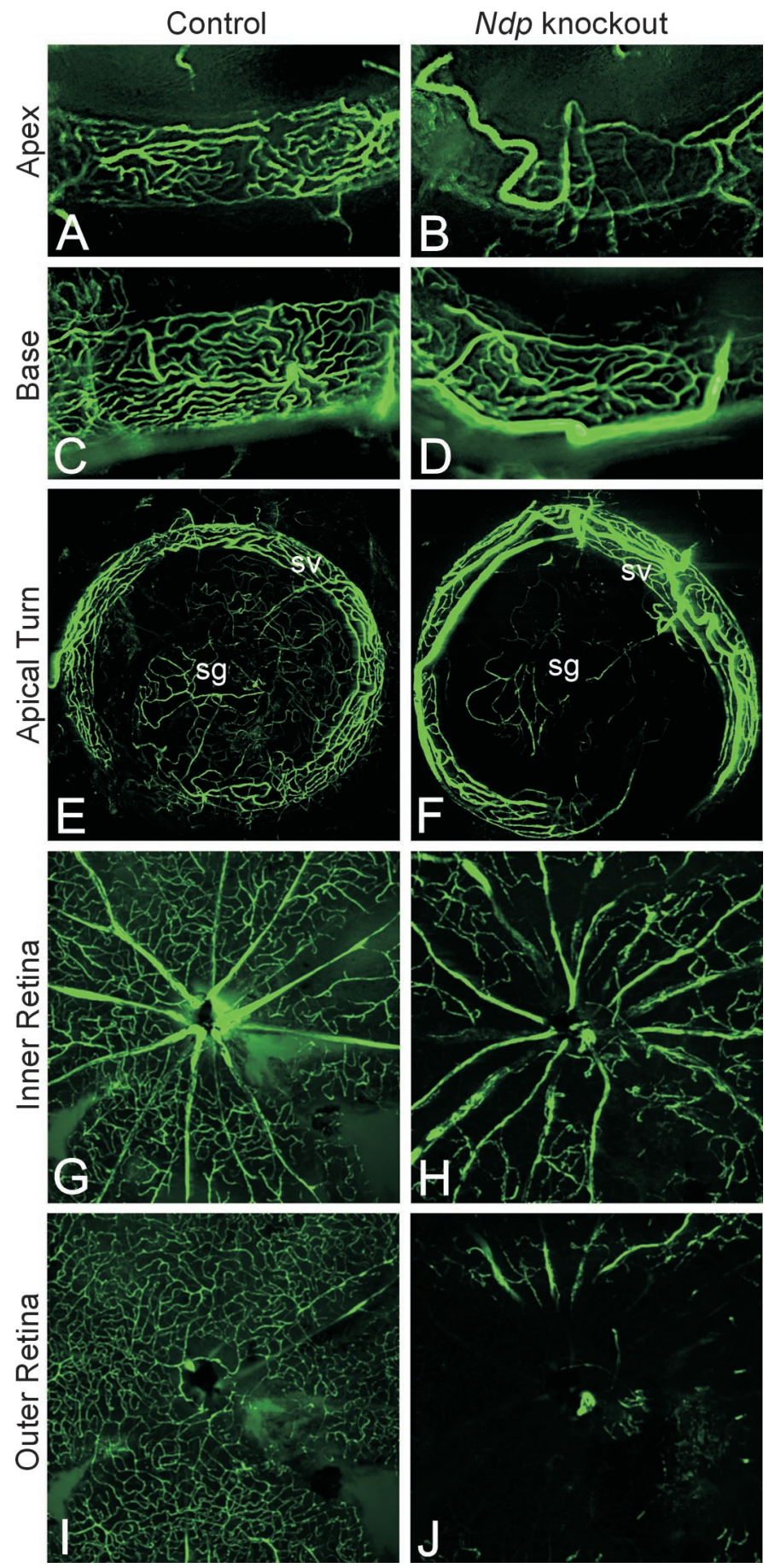

Figure 6. Fluorescent dye fills of cochlear vasculature. $A$, Portion of the apical turn of the stria vascularis in a 2-month-old control mouse. $B$, Apical turn of a 2-month-old knock-out mouse. Note the enlarged capillary on the left and the thin and sparsely distributed vessels on the right. $C$, Basal turn of the stria vascularis in a 2-month-old control mouse. $D$, Basal turn of the stria vascularis in a 2-month-old knock-out mouse. Note the large vessel at the bottom. E, Top view of the apical turn of the cochlea in a 3.5-month-old control mouse. $F$, Similar view of the cochlea in a 3.5-month-out knock-out mouse. Note the large vessels in the stria vascularis $(s v)$ and sparse distribution of vessels in the spiral ganglion $(s g) . G$, Inner retina from a 13 -month-old control mouse. $H$, Inner retina from a 13-month-old knock-out mouse. I, Outer retina from a 13-month-old knock-out mouse. $J$, Outer retina from a 13-month-old knock-out mouse. [The partial vasculature at the top of the image is attributable to a fold from the inner retina (see top of $H$ ).] 
become profoundly deaf (Nadol et al., 1990). Unfortunately, this specimen was not highly informative because of the late stage of disease. Almost all cochlear structures showed degeneration, including the stria vascularis, spiral ganglion, and organ of Corti, reminiscent of that observed in the knock-out mice after 13 months. As a consequence, the study of the knock-out mouse model is the only method available to examine the early stages of the cochlear pathology.

The stria vascularis is the structure most consistently affected in all cochlear regions and at all stages, suggesting that this is the location of the earliest primary lesion. Abnormalities were observed at the earliest stage examined, even in mice with very little hearing loss; these included enlarged vessels and cellular pathology in the stria vascularis (Fig. 4). Pathology in the stria vascularis worsened as the hearing loss progressed.

In addition, loss of outer hair cells was correlated with the rise in auditory threshold. In early stages, the greatest loss was in the basal turn, which is consistent with the more pronounced highfrequency loss. Because the function of outer hair cells is dependent on the endocochlear potential generated by the stria vascularis, loss of outer hair cells is likely to result from strial dysfunction. Supporting this idea is the more significant pathology and hearing loss in the basal turn, which is known to be more susceptible to endocochlear potential abnormalities. In contrast, the inner hair cells were well maintained until the onset of profound deafness.

The neurons of the spiral ganglion also showed a gradual degeneration, leading to severe loss by 15 months of age. Although this pathology was not present as early as the strial pathology, the spiral ganglion may still be affected as a primary consequence of the absence of norrin. The presence of norrin expression in the spiral ganglion neurons, along with an early abnormal vasculature in this region, would support this idea.

Interestingly, at least one human patient with Norrie disease has received a cochlear implant, and it has functioned well for over 12 years since the onset of profound deafness. Thus, the loss of spiral ganglion neurons is apparently not complete in human patients, and the nerves can be maintained for a prolonged period, if not indefinitely, with electrical stimulation.

The primary site of pathology is consistent with the location of $N d p$ gene expression. Predominant expression in the cochlea is in the neurons of the spiral ganglion and marginal cells of the stria vascularis, with minor expression in the spiral ligament (Chen, unpublished observations). Although norrin is a secreted factor and could therefore diffuse and interact with other cell types, protein-binding studies have suggested that it has limited diffusional capacity and may be bound by the extracellular matrix (Perez-Vilar and Hill, 1997; Rehm, unpublished observations). Thus, the site of action of norrin matches the sites of cochlear pathology in Norrie disease.

One of the earliest inner-ear phenotypes observed in knockout mice is an abnormal cochlear vasculature. In particular, the abnormally large vessels and the lack of a well developed capillary bed in both of the two major vascular systems of the cochlea suggest a defect in either the development or maintenance of these systems. Knock-out mice show similarly improper development of the retinal vasculature, including vessels of highly varied sizes, a poorly developed capillary bed, and a lack of penetration of the vessels into the outer layers of the retina (Richter et al., 1998). Furthermore, the cochlear vasculature eventually degenerates, with two-thirds of the vessels lost by 15 months. This may either be secondary to the tissue degeneration in the stria vascu- laris (and decreasing demand for blood supply) or may be attributable to a dysfunctional association of the vasculature with surrounding cells.

A defective interaction between the marginal cells and the vessels could explain the aberrant vascular structure in the stria vascularis. During normal development, the capillary vessels of the stria vascularis grow from the neighboring spiral ligament capillary network (Ando and Takeuchi, 1998; Iwagaki et al., 2000). These strial capillaries are then surrounded by extensions of the marginal and intermediate cells (Anniko and BaggerSjoback, 1984; Takeuchi et al., 2001). Because the marginal cells express $N d p$, norrin may facilitate the interaction between the marginal cells and the capillaries. Without norrin, the cells of the stria vascularis might malf unction because of a diminished ability to acquire nutrients and gases from the vascular system. This would be followed by a shift in the hearing threshold as the endocochlear potential and high potassium concentrations, generated by the stria and needed for hair-cell function, decline (Sewell, 1984).

Interestingly, an additional phenotype that has been associated with Norrie disease is venous insufficiency (Rehm et al., 1997). Although this peripheral vascular disease has been described in only one large kindred, the same phenotype was found recently in an unrelated patient with Norrie disease (Rehm, unpublished observations), suggesting a more direct relationship with Norrie disease mutations. As such, this abnormality of the peripheral vascular system may indicate a more broad role for norrin in the vascular system.

The stria vascularis and possibly the spiral ganglion neurons, and the vascular systems associated with these structures, are apparently the major sites of cochlear dysfunction in Norrie disease. Although the exact function of norrin in the cochlea remains to be elucidated further, the common theme of disrupted vasculature in the ear and the eye, as well as other peripheral sites, implies an important role in basic vascular biology and in the intimate connections a tissue makes with its vascular support.

\section{REFERENCES}

Ando M, Takeuchi S (1998) Postnatal vascular development in the lateral wall of the cochlear duct of gerbils: quantitative analysis by electron microscopy and confocal laser microscopy. Hear Res 123:148-156.

Anniko M, Bagger-Sjoback D (1984) The stria vascularis. In: Ultrastructural atlas of the inner ear (Friedmann I, Ballantyne J, eds), p 329. Boston: Butterworths.

Berger W, Meindl A, van de Pol TJ, Cremers FP, Ropers HH, Doerner C, Monaco A, Bergen AA, Lebo R, Warburg M, Zergollern L, Lorenz B, Gal A, Bleeker-Wagemakers EM, Meitinger T (1992) Isolation of a candidate gene for Norrie disease by positional cloning. Nat Genet 1:199-203.

Berger W, van de Pol D, Bachner D, Oerlemans F, Winkens H, Hameister H, Wieringa B, Hendriks W, Ropers HH (1996) An animal model for Norrie disease (ND): gene targeting of the mouse ND gene. Hum Mol Genet 5:51-59.

Chen ZY, Hendriks RW, Jobling MA, Powell JF, Breakefield XO, Sims KB, Craig IW (1992) Isolation and characterization of a candidate gene for Norrie disease. Nat Genet 1:204-208.

Chen ZY, Battinelli EM, Fielder A, Bundey S, Sims K, Breakefield XO, Craig IW (1993) A mutation in the Norrie disease gene (NDP) associated with X-linked familial exudative vitreoretinopathy. Nat Genet 5:180-183.

Fuchs S, Kellner U, Wedemann H, Gal A (1995) Missense mutation (Arg121Trp) in the Norrie disease gene associated with X-linked exudative vitreoretinopathy. Hum Mutat 6:257-259.

Gorlin RJ, Toriello HV, Cohen MM (1995) Hereditary hearing loss and its syndromes. Oxford: Oxford UP.

Guyer DR, Yannuzzi LA, Chang S, Shields JA, Green WR (1999) Retina-vitreous-macula. Philadelphia: Saunders.

Iwagaki T, Suzuki T, Nakashima T (2000) Development and regression of cochlear blood vessels in fetal and newborn mice. Hear Res 145:75-81.

Meindl A, Berger W, Meitinger T, van de Pol D, Achatz H, Dorner C, 
Haasemann M, Hellebrand H, Gal A, Cremers F, Ropers HH (1992) Norrie disease is caused by mutations in an extracellular protein resembling C-terminal globular domain of mucins. Nat Genet $2: 139-143$.

Meindl A, Lorenz B, Achatz H, Hellebrand H, Schmitz-Valckenberg P, Meitinger T (1995) Missense mutations in the NDP gene in patients with a less severe course of Norrie disease. Hum Mol Genet 4:489-490.

Meitinger T, Meindl A, Bork P, Rost B, Sander C, Haasemann M, Murken J (1993) Molecular modelling of the Norrie disease protein predicts a cystine knot growth factor tertiary structure. Nat Genet 5:376-380.

Nadol Jr JB, Eavey RD, Liberfarb RM, Merchant SN, Williams R, Climenhager D, Albert DM (1990) Histopathology of the ears, eyes, and brain in Norrie's disease (oculoacousticocerebral degeneration). Am J Otolaryngol 11:112-124.

Online Mendelian Inheritance in Man, OMIM (2001) Johns Hopkins University and National Center for Biotechnology Information, MIM number 310600, available at http://www.ncbi.nlm.nih.gov/omim/.

Parving A, Warburg M (1977) Audiological findings in Norrie's disease. Audiology 16:124-131.

Parving A, Elberling C, Warburg M (1978) Electrophysiological study of Norrie's disease: an X-linked recessive trait with hearing loss. Audiology 17:293-298.

Perez-Vilar J, Hill RL (1997) Norrie disease protein (norrin) forms disulfide-linked oligomers associated with the extracellular matrix. J Biol Chem 272:33410-415.

Rehm HL, Gutierrez-Espeleta GA, Garcia R, Jimenez G, Khetarpal U, Priest JM, Sims KB, Keats BJ, Morton CC (1997) Norrie disease gene mutation in a large Costa Rican kindred with a novel phenotype including venous insufficiency. Hum Mutat 9:402-408.

Richter M, Gottanka J, May CA, Welge-Lussen U, Berger W, LutjenDrecoll E (1998) Retinal vasculature changes in Norrie disease mice. Invest Ophthalmol Vis Sci 39:2450-2457.
Sewell WF (1984) The effects of furosemide on the endocochlear potential and auditory-nerve fiber tuning curves in cats. Hear Res 14:305-314.

Shastry BS (1998) Identification of a recurrent missense mutation in the Norrie disease gene associated with a simplex case of exudative vitreoretinopathy. Biochem Biophys Res Commun 246:35-38.

Shastry BS, Hejtmancik JF, Trese MT (1997) Identification of novel missense mutations in the Norrie disease gene associated with one $\mathrm{X}$-linked and four sporadic cases of familial exudative vitreoretinopathy. Hum Mutat 9:396-401.

Takeuchi S, Ando M, Sato T, Kakigi A (2001) Three-dimensional and ultrastructural relationships between intermediate cells and capillaries in the gerbil stria vascularis. Hear Res 155:103-112.

Torrente I, Mangino M, Gennarelli M, Novelli G, Giannotti A, Vadala P, Dallapiccola B (1997) Two new missense mutations (A105T and $\mathrm{C} 110 \mathrm{G})$ in the norrin gene in two Italian families with Norrie disease and familial exudative vitreoretinopathy. Am J Med Genet 72:242-244.

Warburg M (1961) Norrie's disease: a new hereditary bilateral pseudotumor of the retina. Acta Ophthalmol 39:757-772.

Warburg M (1963) Norrie's disease. Acta Ophthalmol 14:134-146.

Warburg M (1965) Norrie's disease. Trans Ophthalmol Soc UK 85:391-408.

Warburg M (1966) Norrie's disease: a congenital progressive oculoacoustico-cerebral degeneration. Acta Ophthalmol Scand Suppl 89:1-47.

Xiang M, Gan L, Zhou L, Klein WH, Nathans J (1996) Targeted deletion of the mouse POU domain gene Brn-3a causes selective loss of neurons in the brainstem and trigeminal ganglion, uncoordinated limb movement, and impaired suckling. Proc Natl Acad Sci USA 93:11950-11955.

Zheng QY, Johnson KR, Erway LC (1999) Assessment of hearing in 80 inbred strains of mice by ABR threshold analyses. Hear Res 130:94107. 